African Journal of Biomedical Research, Vol. 10 (2007); 1 - 8

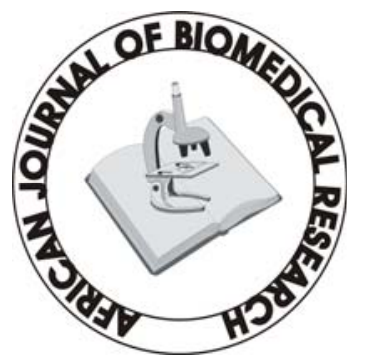

Full-text available at www.ajbrui.com www.bioline.br/md www.ajol.com
Received:

October, 2006

Accepted

(Revised):

December, 2006

Published

January, 2007
Review Article

\section{Essential Hypertension: Another Look at Possible Aetiology and Treatment}

\author{
M.I. Ebomoyi* and V.I. Iyawe \\ Department of Physiology, College of Medical Sciences, \\ University of Benin, Benin City, Nigeria.
}

\begin{abstract}
Hypertension is a world - wide problem with sometimes very devastating complications especially in the black race. It is a silent disease and a silent killer through its assault on the vital organs of the body like the heart, brain and kidneys. There are well known therapeutic modalities, including diet and weight control, regular exercise and other lifestyle modifications like cessation of smoking and moderation in alcohol intake. Of course, the beneficial effect of drugs in the control of hypertension is well established. This review addresses the physiologic and genetic basis for the evaluation and management of hypertension and for maximizing individual treatment needs. Genes responsible for inherited essential hypertension can be targeted and hypertension effectively prevented or treated. A pharmacogenomic approach to the treatment of hypertension is proposed.
\end{abstract}

(Afr. J. Biomed. Res. 10: 1 - 8, January 2007)

Keywords: Hypertension, Genes, Physiology, Therapy

*Address for Correspondence: e- mail: maureenebomoyi@yahoo.com;

$2348023396806 ; 2348059239875$.

Abstracted by:

African Index Medicus (WHO), CAB Abstracts, Index Copernicus, Global Health Abstracts, Asian Science Index, Index Veterinarius, Bioline international, African Journals online 


\section{INTRODUCTION}

High blood pressure (BP), otherwise known as hypertension is a major health problem especially among blacks (Animah, 2004). A dangerous aspect of hypertension is that someone can feel healthy and still have it. So, the most that can be done to detect it is through a diagnosis made by a doctor, using a blood pressure monitor. From our daily routine, habits and genetic heritage, many factors can result in high blood pressure, the silent disease that leads to heart attack, stroke, and kidney failure in adults. The disease is rapidly becoming a concern in children (Peck, 2004). High BP is a heritable trait (Adeyemo et al, 2002), but the identification of susceptibility genes involved in the pathophysiology has previously been a subject of much controversy (Swales, 1985). In recent times, researches are beginning to tally regarding the genetic basis of this condition (Timberlake et al, 2001; Yagil and Yagil, 2005). This review of the physiological basis of the therapeutic modalities that can be employed when essential hypertension is genetically identified is intended to aid the clinician in identifying those individuals at high risk who need to undergo evaluation and treatment, as well as in selecting optimal treatment strategies for hypertensive patients that are tailored towards their individual needs. Information gained from the human genome project and from studies of the genetic basis of hypertension both in animal models and human populations may revolutionize the treatment of hypertension by replacing current empirical therapy with more effective, targeted treatments based on the genotype of the patient - the socalled 'pharmacogenomic' approaches to antihypertensive therapy.

\section{Essential or Primary Hypertension}

$\mathrm{BP}$ is a quantitative trait that is highly variable (Parati et al, 1998). There is a strong positive and continuous correlation between BP and the risk of cardiovascular disease (CVD), renal disease, and mortality. This correlation is more robust with systolic than with diastolic BP (PastoBarriuso et al, 2003). There is no specific level of
BP where cardiovascular or renal complications start to occur; thus the definition of hypertension is arbitrary but needed for practical reasons in patient assessment and treatment (Carretero and Oparil, 2000). The Sixth Report of the Joint National Committee on Prevention, Detection, Evaluation, and Treatment of High Blood Pressure (JNC VI, 1997) defined and classified hypertension in adults, as shown in Table1.

Table 1:

Definitions and Classification of Blood Pressure Levels

\begin{tabular}{lll}
\hline Category & $\begin{array}{l}\text { Systolic, } \\
\text { mmHg }\end{array}$ & $\begin{array}{l}\text { Diastolic, } \\
\text { mmHg }\end{array}$ \\
\hline Optimal & $<120$ & and $<80$ \\
\hline Normal & $<130$ & and $<85$ \\
\hline High normal & $130-139$ & or $85-89$ \\
\hline $\begin{array}{l}\text { Hypertension: } \\
\text { Stage 1 (mild) } \\
\text { (borderline) }\end{array}$ & $140-159$ & or $90-99$ \\
Stage 2 (moderate) & $140-149$ & or $90-94$ \\
Stage 3 (severe) & $160-179$ & or $100-109$ \\
& $\geq 180$ & or $\geq 110$ \\
& & \\
\hline $\begin{array}{l}\text { Isolated systolic } \\
\text { hypertension } \\
\text { Subgroup: borderline }\end{array}$ & $140-149$ & and $<90$ \\
\hline
\end{tabular}

When a patient's systolic and diastolic blood pressures fall into different categories, the higher category should apply (JNC VI, 1997).

Essential, primary or idiopathic hypertension is defined as high BP in which secondary causes such as renovascular disease, renal failure, pheochromocytoma, aldosteronism, or other causes of secondary hypertension or mendelian forms (monogenic) are not present. Essential hypertension accounts for $95 \%$ of all cases of hypertension. It is a heterogenous disorder, with different patients having different causal factors that lead to high BP. Essential hypertension needs to be separated into various syndromes because the causes of high BP in most patients presently classified as having essential 
hypertension can be recognized.

The genetic alterations responsible for inherited 'essential' hypertension remain largely unknown. Jeunemaitre et al (1997) first reported a polymorphism in the angiotensinogen gene linked with essential hypertension in hypertensive siblings from Utah and France. Polymorphisms and mutations in other genes such as angiotensin-converting enzyme, $\beta_{2^{-}}$ adrenergic receptor, $\alpha$-adducin, angiotensinase C, rennin-binding protein, G-protein $\beta_{3}$-subunit, atrial natriuretic factor, and the insulin receptor have also been linked to the development of essential hypertension; however, most of them show a weak association if any, and most of these studies need further confirmation.

\section{Known Etiological Factors}

Although it has frequently been indicated that the causes of essential hypertension are not known, this is only partially true because we have little information on genetic variations or genes that are over-expressed or under-expressed as well as the intermediary phenotypes that they regulate to cause high BP (Luft, 1998). A number of factors are known to increase BP, including (1) obesity, (2) insulin resistance, (3) high alcohol intake, (4) high salt intake (in salt-sensitive patients), (5) aging (6) sedentary lifestyle, (7) stress, (8) low potassium intake, and (9) low calcium intake (Sever, 1989). Furthermore, many of these factors are additive, such as obesity and alcohol intake. Variations in BP that are genetically determined are called 'inherited BP' (Carretero and Oparil, 2000), and family studies have shown that inherited BP can range from low normal BP to severe hypertension. Factors that increase BP, such as obesity, insulin resistance and high alcohol and salt intake are called 'hypertensinogenic factors'. Some of these factors have inherited, behavioral, and environmental components.

Recognition of the hypertensinogenic factors may allow non-pharmacological intervention. Hypertensinogenic factors have an important genetic component. Furthermore, there are interactions between genetic and environmental factors, which in turn influence intermediary phenotypes such as sympathetic nerve activity, the renin-angiotensin-aldosterone and renal kallikrein-kinin systems, and endothelial factors, which in turn influence other intermediary phenotypes such as sodium excretion, vascular reactivity, and cardiac contractility. These and many other intermediary phenotypes determine total vascular resistance and cardiac output and, consequently, BP. Recognition of the hypertensinogenic factor(s) and establishing that the patient's hypertension is the result of obesity (either alone or combined with other factors such as insulin resistance or high alcohol intake) or old age instead of essential hypertension may help the physician as well as the patient and his or her family to modify or eliminate these hypertensinogenic and CVD risk factors when possible, which may cure the hypertension or at least facilitate control of BP. When the hypertensinogenic factor cannot be reduced or eliminated, as with systolic hypertension induced by aging (arteriosclerosis), recognition of the underlying cause of high BP will emphasize the need for (1) further studies to determine whether the patient has arteriosclerosis and / or atherosclerosis, the magnitude of the disease, and whether there are occlusive lesions; (2) treatment of the atherosclerosis with lifestyle and dietary changes and lipid - lowering agents if necessary; and (3) pharmacological treatment of systolic hypertension to decrease passive stiffness (arteriosclerosis) of the major central elastic arteries and decrease morbidity and mortality rates. Thus recognition of factors that induce hypertension is not only of theoretical but also of practical importance. In summary therefore, it is no longer appropriate to define essential hypertension as a rise in blood pressure without cause, since a number of causes can be clearly identified in most cases of so-called 'essential hypertension'

\section{Inherited BP}

The influence of genes on BP has been suggested by family studies demonstrating associations of BP among siblings and between parents and children. Genetic factors also influence behavioral patterns, which might lead 
to BP elevation. For example, a tendency toward obesity or alcoholism will be influenced by both genetic and environmental factors.

Mutations in at least 10 genes have been shown to raise or lower BP through a common pathway by increasing or decreasing salt and water reabsorption by the nephron (Lifton, 1996). The genetic mutations responsible for 3 rare forms of mendelian (monogenic) hypertensive syndromes - glucocorticoid- remediable aldosteronism (GRA), Liddle's syndrome, and apparent mineralocorticoid excess - have been identified, whereas in a fourth, autosomal dominant hypertension with brachydactyly, the gene is not yet identified but has been mapped to chromosome 12 (12p). Subtle variations in one of these genes may also cause some forms of 'essential' hypertension.(Lifton, 1996).

Glucocorticoid - Remediable Aldosteronism This is an autosomal dominant form of monogenic hypertension in which aldosterone secretion is regulated by adrenocorticotropic hormone. Glucocorticoid treatment causes BP to decrease and gives the syndrome its name. The genetic mutation that causes GRA has been identified by Lifton (1995) as a chimeric gene fusing nucleotide sequences of the promoter regulatory region of $11 \beta$-hydroxylase (controlled by adrenocorticotropic hormone) and the structural portion of the aldosterone synthase gene. The patients are usually thought to have primary aldosteronism because they exhibit volume expansion, metabolic alkalosis with hypokalemia, low plasma renin, and high aldosterone. However, with the development of direct genetic testing, the BP of patients with this syndrome has been found to cover a wide range, including normotensive levels (Gates et al, 1996). The BP would be the combined result of the inherited BP (including the genetic mutation) and the increase in BP caused by hypertensinogenic factors such as salt.

\section{Liddle's Syndrome}

This is an autosomal dominant form of monogenic hypertension that results from mutations in the amiloride - sensitive epithelial sodium channel, leading to increased channel activity (Findling et al, 1997). The mutations reported to date result in the elimination of 45 to 75 amino acids from the cytoplasmic carboxyl terminus of $\beta$ - or $\gamma$ - subunits of the channel; thus Liddle's syndrome is genetically heterogenous. It is characterized by the early onset of hypertension with hypokalemia and suppression of both plasma renin activity and aldosterone, the latter differentiating this syndrome from primary aldosteronism. Both the hypertension and the hypokalemia vary in severity, raising the possibility that some patients classified as having salt - sensitive essential hypertension actually have Liddle's syndrome (Hansson et al, 1995). It is also possible that high $\mathrm{BP}$ in blacks, who are frequently salt-sensitive, is due to a polymorphism in one of the sodium channel genes or in one of the genes of systems that regulate it, causing its activity to increase.

\section{Apparent Mineralocorticoid Excess}

This is an autosomal recessive form of monogenic juvenile hypertension that results from a mutation in the renal-specific isoform $11 \beta$-hydroxysteroid dehydrogenase gene (Mune et al, 1995). Normally this enzyme converts cortisol to the inactive metabolite cortisone. In the distal nephron, this is important because cortisol and aldosterone have a similar affinity for the mineralocorticoid receptor. The enzymatic deficiency allows the mineralocorticoid receptors in the nephron to be occupied and activated by cortisol, causing sodium and water retention, volume expansion, low rennin, low aldosterone, and more importantly, a salt-sensitive form of hypertension. Thus this gene may be a locus for salt-sensitive essential hypertension.

\section{Autosomal Dominant Hypertension with Brachydactyly}

In this monogenic syndrome, hypertension and brachydactyly are always inherited together (100\% co-segregation) (Luft, 1998). Affected persons are shorter than non-affected relatives. The gene for hypertension has been mapped to the short arm of chromosome 12 (12p) in a 
Turkish kindred, although the gene responsible for this syndrome has not yet been cloned. Unlike the other 3 autosomal forms of hypertension, BP is not affected by volume expansion and the underlying mechanism is not known. Thus, identification of the gene responsible may help clarify some of the genetic alterations in this form of essential hypertension.

\section{HYPERTENSINOGENIC FACTORS}

There is evidence that obesity, insulin resistance, high alcohol intake, high salt intake, a sedentary lifestyle, stress, dyslipidemia, and low potassium or calcium intake increase BP in susceptible subjects.

\section{Obesity and Insulin Resistance}

Obesity, and especially abdominal obesity, is the main hypertensinogenic factor. Obesity is also the cause of insulin resistance, adult - onset diabetes mellitus, left ventricular hypertrophy, hyperlipidemia, and atherosclerotic disease. A BMI of $<25$ is considered normal or healthy, whereas a BMI of 26 to 28 increases the risk of high BP by $180 \%$ and the risk of insulin resistance by $>1000 \%$. Thus insulin resistance is present in many patients with obesity and hypertension.

The mechanism by which obesity raises BP is not fully understood, but increased BMI is associated with an increase in plasma volume and cardiac output; both these alterations and BP can be decreased by weight loss in both normotensive and hypertensive subjects (Rocchini et al, 1989), even when sodium intake is kept relatively constant (Reisin et al, 1978). Exercise decreases plasma insulin by a different mechanism than loss of body weight. Furthermore, non-obese hypertensive subjects are much more likely to exhibit insulin resistance than normotensive individuals ( Modan et al, 1985). Insulin by itself has a vasodilator effect. Insulin - like growth factor I (Diez, 1999), and leptin, a neuropeptide that regulates appetite, have also been implicated in the pathogenesis of obesity - induced hypertension. Thus, although the mechanisms by which obesity and insulin resistance increase BP remain undefined, it is clear that these increases in $\mathrm{BP}$ are overlain on the inherited BP.

\section{THERAPEUTIC MODALITIES}

Diet and lifestyle modifications may lower BP and obviate the need for drug treatment or reduce the dosages of antihypertensive drugs needed to control BP. A reasonable generalized approach for all patients includes (1) weight loss for the overweight patient; (2) regular physical activity; (3) moderation of alcohol consumption; (4) dietary modification to reduce sodium and fat and increase calcium, potassium, magnesium, vitamins, and fiber from food sources; and (5) cessation of smoking. Such an approach has been shown to produce significant sustained reductions in BP while reducing overall cardiovascular risk. The same lifestyle modifications that are effective in treating hypertensive patients may be useful in the primary prevention of essential hypertension.

\section{Weight Reduction}

Weight loss is closely correlated with reduction in BP and appears to be the most effective of all non-pharmacological measures used to treat hypertension. Weight loss also enhances the efficacy of antihypertensive drugs. This effect is independent of dietary sodium restriction and is seen in both obese and non-obese hypertensive individuals.

Weight reduction of at least $10 \mathrm{Ib}$ (with further increments depending on the initial response and the patient's baseline weight) through a combination of dietary caloric restriction and increased physical activity is recommended for all overweight hypertensive individuals (WHO, 1999).

\section{Increased Physical Activity}

Studies suggest that at least 30 minutes of moderately intense physical activity such as brisk walking, swimming, bicycling, or yard work preferably once per week, can lower BP. Isometric exercise such as heavy weight lifting 
can have a pressor effect and should be avoided particularly in patients with advanced or unstable CVD.

\section{Moderation of Alcohol Intake}

Alcohol consumption elevates BP both acutely and chronically. The effect increases with age, is independent of the type of alcoholic beverage, and is additive but independent of the effects of obesity, oral contraceptives, and high salt intake (Arkwright et al, 1982). However, moderate alcohol consumption ( $<3$ standard drinks a day) reduces overall CVD risk in the general population (Emberson et al, 2005). Whether this risk reduction also occurs in the hypertensive population needs to be studied further. The mechanism by which alcohol raises BP is not known. Excessive alcohol intake also appears to cause resistance to antihypertensive therapy.

\section{Dietary Modification}

The Dietary Approaches to Stop Hypertension (DASH) trial showed overall reductions in BP of 11.4 / $5.5 \mathrm{mmHg}$ in hypertensive persons on a diet rich in fruits, vegetables, and low-fat dairy products, compared with control subjects, while dietary sodium intake and weight were held constant. This reduction in BP was independent of race, sex, age, BMI, level of education, income, physical activity, family history of hypertension, and geographic location of the study site (Svetkey et al, 1999). The success of the DASH diet in lowering BP cannot be attributed solely to its micronutrient content (high in calcium, potassium, and magnesium) because the foods included in the DASH trial contain complex combinations of minerals, macronutrients, fiber, phytochemicals, vitamins, and other factors that alone or in combination could lower BP (Azadbakht et al, 2005). Translation of the results of the DASH trial to advice for the general public or the universe of hypertensive patients is more easily accomplished by recommending 4 servings of fruit, 4 servings vegetables, and 3 servings of low-fat dairy products per day than by prescribing a specific daily intake of calcium, potassium, and magnesium. The paradigm shift toward recognition of the powerful role of total diet (rather than individual nutrients) in the prevention and treatment of hypertension in particular and CVD in general deserves emphasis.

\section{Pharmacological Treatment}

Reducing BP by pharmacological means clearly reduces CVD morbidity and mortality rates. ACE inhibitors, used alone or in combination with diuretics and digoxin, both prevent congestive heart failure and reduce morbid and mortal events in patients with established failure. Clinical trials have also shown benefit from appropriately dosed $\beta$ - blockers in setting heart failure (MERIF-HF, 1999). Alternative therapies include hydralazine and isosorbide dinitrate as well as the dihydropyridine calcium channel blockers amlodipine and felodipine. ACE inhibitors are recommended as first - line agents for treating hypertension in diabetic patients, particularly patients with type 1 diabetes and /or diabetic nephropathy, because they reduce proteinuria and slow the rate of deterioration in renal function. Other classes of antihypertensive drugs are needed in diabetics. Because of the aggressive nature of target organ damage in diabetics, a lower BP goal ( $<130$ / $85 \mathrm{mmHg})$ is recommended, usually necessitating a multidrug regimen.

$\beta$-Blockers and some long - acting calcium channel blockers have anti-angina properties, whereas $\beta$-blockers are useful in the secondary prevention of acute myocardial infarction and sudden cardiac death (Pitt et al, 1999). The utility of ARBs in these conditions is not yet clear.

\section{CONCLUSION}

It is concluded that efforts to identify hypertension - predisposing genetic loci have focused largely on candidate gene strategies, in which specific candidates have been tested for linkage and association with blood pressure or the diagnosis of hypertension. These studies, including several genome - wide scans, have yielded highly promising results suggesting a number of potential candidate and genomic 
regions that may contribute to blood pressure variation. However, how these genes fit into the complex pathophysiological network that induces hypertension remains unclear.

Identification of the constellation of genes responsible for inherited essential hypertension will yield even more targeted and effective preventive and therapeutic strategies. This may occur through specific lifestyle modifications directed toward persons at high risk for CVD because of their genotype, made possible by more selective, genotype - targeted 'pharmacogenomic' treatments or even gene therapy.

\section{REFERENCES}

Adeyemo, A.A., Omotade, O.O., Rotimi, C.N., Luke, A.H., Tayo, B.O., Cooper, R.S. (May 2002): Heritability of blood pressure in Nigerian families. J. Hypertens. 20(5), $859-863$.

Animah R. (August 2004): Hypertension epidemic. Community / Health. HarlemLIVE Internet Publication By The Youth of NYC.

Arkwright, P.D., Beilin, L.J., Rouse, I., Armstrong, B.K., Vandongen, R. (1982): Effects of alcohol use and other aspects of lifestyle on blood pressure levels and prevalence of hypertension in a working population. Circulation. 66, $60-66$.

Azadbakht, L., Mirmiran, P., Esmaillzadeh, A., Azizi, T., Azizi, F. (2005): Beneficial effects of a dietary approach to stop hypertension eating plan on features of the metabolic syndrome. Diabetes Care. 28, $2823-2831$.

Carretero, O.A., Oparil, S. (2000): Essential hypertension, Part 1: Definition and Etiology. Circulation. 101, 329 - 335.

Corvol, P., Lalouel, J.M. (1997): Haplotypes of angiotensinogen in essential hypertension. Am. J. Hum. Genet. 60, 1448 - 1460.

Diez, J. (1999): Insulin - like growth factor I in essential hypertension. Kidney Int. 55, $744-759$.

Emberson, J.R., Shaper, A.G., Wamanethee, S.G., Morris, R.W., Whinicup, P.H. (2005): Alcohol intake in middle age and risk of cardiovascular diseases and mortality. Am J Epid. 161, 856 - 863.

Findling, J.W., Raff, H., Hansson, J.H., Lifton, R.P. (1997): Liddle's syndrome: prospective genetic screening and suppressed aldosterone secretion in an extended kindred. J. Clin. Endocrinol. Metab. 82, 1071 - 1074 .
Gates, L.J., MacConnachie, A.A., Lifton, R.P., Haites, N.E., Benjamin, N. (1996): Variation of phenotype in patients with glucocorticoid remediable aldosteronism. J. Med. Genet. 33, 25 - 28.

Hansson, J.H., Nelson-Williams, C., Suzuki, H., Schild, L., Shimkets, R., Lu, Y., Canessa, C., Iwasaki, T., Rossier, B., Lifton, R.P. (1995): Hypertension caused by a truncated sodium channel gamma subunit: genetic heterogeneity of Liddle syndrome. Nat. Genet. 11, 76 - 82.

Jeunemaitre, X., Inoue, I., Williams, C., Charru, A., Tichet, J., Powers, M., Sharma, A.M., GimenezRoqueplo, A.P., Hata, A., (1997): Joint National Committee on Prevention, Detection, Evaluation, and Treatment of High Blood Pressure: The Sixth Report of the Joint National Committee on Prevention, Detection, Evaluation, and Treatment of High Blood Pressure (JNC VI). Arch. Intern. Med. 157, 2413 2446.

Lifton, R.P. (1995): Genetic determinants of human hypertension. Proc. Natl. Acad. Sci. U.S.A. 92, 8545 8551.

Lifton, R.P. (1996): Molecular genetics of human blood pressure variation. Science. 272, 676 - 680 .

Luft, F.C. (1998): Molecular genetics of human hypertension. J. Hypertens. 16, 1871 - 1878.

MERIT-HF Study Group. (1999): Effect of Metoprolol CR/XL in chronic heart failure: Metoprolol CR/XL Randomised Intervention Trial in Congestive Heart Failure (MERIT-HF). Lancet. 353, 2001 - 2007.

Modan, M., Halkin, H., Almog, S., Lusky, A., Eshkol, A., Shefi, M., Shitrit, A., Fuchs, Z. (1985): Hyperinsulinemia: a link between hypertension obesity and glucose intolerance. J. Clin. Invest. 75, 809 - 817.

Mune, T., Rogerson, F.M., Nikkila, H., Agarwal, A.K., White, P.C. (1995): Human hypertension caused by mutations in the kidney isozyme of 11 betahydroxysteroid dehydrogenase. Nat. Genet. 10, $394-$ 399.

Parati, G., Di Rienzo, M., Ulian, L., Santucciu, C., Girard, A., Elghozi, J.L., Mancia, G. (1998): Clinical relevance of blood pressure variability. J. Hypertens. 16(3), S25 - S33.

Pasto-Barriuso, R., Beinegas, J.R., Damian, J., Appel, L.J., Guallar, E. (2003): Systolic Blood Pressure, Diastolic Blood Pressure, and Pulse Presure: An evaluation of the joint effect on mortality. Ann. Intern. Med. 139(9), 731 - 739.

Peck, P. (May 2004): Check blood pressure starting at age 3. WebMD Medical News. Medical News Archive. May 20. 
Pitt, B., Zannad, F., Remme, W.J., Cody, R., Castaigne, A., Perez, A., Palensky, J., Wittes, J., for the Randomized Aldactone Evaluation Study Investigators. (1999): The effect of spirinolactone on morbidity and mortality in patients with severe heart failure. N Engl J Med. 341, 709 - 717.

Reisin, E., Abel, R., Modan, M., Silverberg, D.S., Eliahou, H.E., Modan, B. (1978): Effect of weight loss without salt restriction on the reduction of blood pressure in overweight hypertensive patients. N. Engl. J. Med. 298, 1 - 6.

Rocchini, A.P., Key, J., Bondie, D., Chico, R., Moorehead, C., Katch, V., Martin, M. (1989): The effect of weight loss on the sensitivity of blood pressure to sodium in obese adolescents. N. Engl. J. Med. 321, 580 - 585.

Sever, P.S. and Poulter, N.R. (1989): A hypothesis for the pathogenesis of essential hypertension: the initiating factors. J.Hypertens. 7(1), S9 - S12.

Svetkey, L.P., Simons-Morton, D., Vollmer, W.M., Appel, L.J., Conlin, P.R., Ryan, D.H., Ard, J., Kennedy, B.M., for the DASH Research Group. (1999): Effects of dietary patterns on blood pressure: subgroup analysis of the Dietary Approaches to Stop
Hypertension (DASH) randomized clinical trial. Arch Intern Med. 159, 285 - 293.

Swales, J.D. (1985): Platt versus Pickering: An episode in recent medical history. London: Keynes Press (pub.).

Timberlake, D.S., O’Connor, D.T., Parmer, R.J. (2001): Molecular genetics of essential hypertension: recent results and emerging strategies. Curr. Opin. Nephrol. Hypertens. 10(1), 71 - 79.

World Health Organization (1999): International Society of Hypertension guidelines for the management of hypertension. Guidelines Subcommittee. J Hypertens. 17, 151 - 183.

Yagil, Y and Yagil, C. (March 2005): The search for the genetic basis of hypertension. Curr. Opin. Nephrol. Hypertens. 14(2), 141 - 147. 